Pacific Journal of Mathematic 


\title{
SOME PERTURBED ELECTROSTATIC FIELDS
}

\author{
G. Power
}

Summary. When a dielectric body is placed in an electrostatic field, the field becomes perturbed, and the boundary of the dielectric experiences a mechanical force due to the refraction of the lines of force. This paper is concerned with finding expressions for the resultant mechanical force at a surface of discontinuity separating two media of different specific inductive capacities, and also with determining the perturbed fields in both dielectrics (assumed homogeneous and isotropic) for various boundary shapes. In the first section, some two-dimensional fields are discussed, starting with a concentric circular cylindrical system, and then proceeding to other cases. The second section is devoted to an application of Stokes' stream function to three-dimensional axisymmetric fields.

\section{TwO-Dimensional Field}

1.1. Mechanical force and couple. Consider a nested system of homogeneous isotropic dielectric cylinders, and let the perturbed electrostatic fields in the various media be expressed in terms of complex potential functions. Taking the origin of coordinates inside the contour of the interface separating the medium $(s)$ of dielectric constant $k_{s}$ from the medium $(s+1)$ of dielectric constant $k_{s+1}$, we know that the interface $C$ will experience a force whose components are given by [4]

$$
Y+i X=\frac{k_{s+1}}{8 \pi} \int_{C}\left(\frac{d w_{s}+1}{d z}\right)^{2} d z-\frac{k_{s}}{8 \pi} \int_{C}\left(\frac{d w_{s}}{d z}\right)^{2} d z,
$$

where $w_{s+1}$ is the complex potential in the medium $(s+1)$, and $w_{s}$ that in the medium $(s)$.

The anticlockwise moment about the origin of the mechanical forces is

$$
\Gamma=R \frac{k_{s+1}}{8 \pi} \int_{C}\left(\frac{d w_{s}+1}{d z}\right)^{2} z d z-R \frac{k_{s}}{8 \pi} \int_{C}\left(\frac{d w_{s}}{d z}\right)^{2} z d z
$$


1.20. System of two concentric dielectric cylinders. Suppose we have an inner cylinder of radius $b$, dielectric constant $k_{1}$, and a concentric cylinder of radius $a(>b)$, dielectric constant $k_{2}$. Let this system be placed with the common center at the origin of coordinates in a medium of dielectric constant $k_{3}$, in which there is a field whose unperturbed complex potential is

$$
w=\sum_{n=1}^{p} a_{3, n} z^{n}
$$

Suppose there is also a line charge of strength $m$ per unit length embedded in the medium $k_{2}$ at $z=z_{0}$. The perturbed fields can be taken to be

$$
\begin{aligned}
& w_{1}=\sum_{n=1}^{\infty} a_{1, n} z^{n}, \\
& w_{2}=\Omega+\sum_{n=1}^{\infty} a_{2, n} z^{n}+\sum_{n=1}^{\infty} b_{2, n} z^{-n}, \\
& w_{3}=\sum_{n=1}^{p} a_{3, n} z^{n}+\sum_{n=0}^{\infty} b_{3, n} z^{-n}+c_{3} \log z,
\end{aligned}
$$

where (omitting the constant term)

$$
\Omega=-\frac{2 m}{k_{2}} \log \left(z-z_{0}\right)= \begin{cases}\frac{2 m}{k_{2}} \sum_{n=1}^{\infty} \frac{z^{n}}{n z_{0}^{n}}, & \text { if }\left|\frac{z}{z_{0}}\right|<1, \\ \frac{2 m}{k_{2}} \sum_{n=1}^{\infty} \frac{z_{0}^{n}}{n z^{n}}-\frac{2 m}{k_{2}} \log z, \text { if }\left|\frac{z_{0}}{z}\right|<1 .\end{cases}
$$

The boundary conditions are

$$
R\left(w_{1}\right)=R\left(w_{2}\right), k_{1} d\left(w_{1}\right)=k_{2} d\left(w_{2}\right) \text { on }|z|=b \text {, }
$$

and

$$
R\left(w_{2}\right)=R\left(w_{3}\right), k_{2} d\left(w_{2}\right)=k_{3} d\left(w_{3}\right) \text { on }|z|=a \text {. }
$$


Applying these boundary conditions and solving, we obtain

$$
\begin{aligned}
& \alpha_{n} a_{1, n}=4 k_{2} k_{3} a^{2 n} a_{3, n}+\frac{4 m}{n z_{0}^{n}}\left\{\left(k_{2}+k_{3}\right) a^{2 n}+\left(k_{2}-k_{3}\right) z_{0}^{n} \bar{z}_{0}^{n}\right\}, \\
& \alpha_{n} a_{2, n}=2\left(k_{1}+k_{2}\right) k_{3} a^{2 n} a_{3, n} \\
& +\frac{2 m}{n k_{2} z_{0}^{n}}\left(k_{2}-k_{3}\right)\left\{\left(k_{1}+k_{2}\right) z_{0}^{n} \bar{z}_{0}^{n}+\left(k_{2}-k_{1}\right) b^{2 n}\right\}, \\
& a_{n} b_{2, n}=2\left(k_{2}-k_{1}\right) k_{3} a^{2 n} b^{2 n} \bar{a}_{3, n} \\
& +\frac{2 m}{n k_{2} \bar{z}_{0}^{n}}\left(k_{2}-k_{1}\right)\left\{\left(k_{2}+k_{3}\right) a^{2 n}-z_{0}^{n} \bar{z}_{0}^{n}\left(k_{3}-k_{2}\right)\right\} b^{2 n}, \\
& \alpha_{n} b_{3, n}=\left\{\left(k_{3}-k_{2}\right)\left(k_{2}+k_{1}\right) a^{2 n}+\left(k_{3}+k_{2}\right)\left(k_{2}-k_{1}\right) b^{2 n}\right\} a^{2 n} \bar{a}_{3, n} \\
& +\frac{4 m}{n \bar{z}_{0}^{n}}\left\{\left(k_{2}-k_{1}\right) b^{2 n}+\left(k_{2}+k_{1}\right) z_{0}^{n} \bar{z}_{0}^{n}\right\} a^{2 n}, \\
& b_{3,0}=\frac{m\left(k_{2}-k_{3}\right)}{k_{2} k_{3}} \log a^{2}, \quad c_{3}=-\frac{2 m}{k_{3}},
\end{aligned}
$$

where

$$
\alpha_{n}=\left(k_{3}+k_{2}\right)\left(k_{2}+k_{1}\right) a^{2 n}+\left(k_{3}-k_{2}\right)\left(k_{2}-k_{1}\right) b^{2 n} \text {. }
$$

From these results we can easily deduce [5] the perturbed fields when a circular dielectric cylinder is introduced into the general field $w=f(z)$.

1.21. Special cases of the complex potential. If in 1.20 the cylinder of radius $b$ is made a conducting surface, then $k_{1}=\infty$. Putting also

$$
k_{3}=1, k_{2}=k, m=0, a_{3, n}=E_{n},
$$

we obtain 


$$
\begin{aligned}
a_{2, n} & =\frac{2 a^{2 n} E_{n}}{(k+1) a^{2 n}+(k-1) b^{2 n}}=\frac{2 E_{n}}{(1+k)}\left\{1+\frac{\lambda b^{2 n}}{a^{2 n}}+\cdots+\frac{\lambda^{p} b^{2 n p}}{a^{2 n p}}+\cdots\right\}, \\
b_{2, n} & =\frac{-2 a^{2 n} b^{2 n} \bar{E}_{n}}{(k+1) a^{2 n}+(k-1) b^{2 n}}=-\frac{2 \bar{E}_{n} b^{2 n}}{(1+k)}\left\{1+\frac{\lambda b^{2 n}}{a^{2 n}}+\cdots+\frac{\lambda^{p} b^{2 n p}}{a^{2 n p}}+\cdots\right\}, \\
b_{3, n} & =-\frac{\left\{(k-1) a^{2 n}+(k+1) b^{2 n}\right\} a^{2 n} \bar{E}_{n}}{(k+1) a^{2 n}+(k-1) b^{2 n}} \\
& =\bar{E}_{n} a^{2 n}\left\{\lambda+\left(\lambda^{2}-1\right) \frac{b^{2 n}}{a^{2 n}}+\cdots\left(\lambda^{p+1}-\lambda^{p-1}\right) \frac{b^{2 n p}}{a^{2 n p}}+\cdots\right\},
\end{aligned}
$$

where $\lambda=(1-k) /(1+k)$.

From these expansions we observe that if this system is placed at the origin of coordinates in a field whose unperturbed form in air is

$$
f(z)=\sum_{n=1}^{p} E_{n} z^{n}
$$

then the complex potentials in air and in the dielectric become respectively

$$
w_{0}=f(z)+\lambda \bar{f}\left(a^{2} / z\right)+\sum_{p=1}^{\infty}\left(\lambda^{p+1}-\lambda^{p-1}\right) \bar{f}\left(b^{2 p} / z a^{2 p-2}\right),
$$

and

$$
w_{i}=\frac{2}{(1+k)} \sum_{p=0}^{\infty} \lambda^{p}\left\{f\left(\frac{z b^{2 p}}{a^{2 p}}\right)-\bar{f}\left(\frac{b^{2 p+2}}{z a^{2 p}}\right)\right\} .
$$

Application of the theory of summation of double series shows that (1), (2) above still hold if $f(z)$ is an infinite series assumed regular in a region including $|z| \leq a$.

If now we require $|z|=b$ to be a line of force instead of a conducting surface, we set $k_{1}=0$ instead of $k_{1}=\infty$. Proceeding as above, we obtain the perturbed fields for this new system in the form 


$$
\begin{aligned}
& w_{0}=f(z)-\mu \bar{f}\left(\frac{a^{2}}{z}\right)-\sum_{p=1}^{\infty}\left(\mu^{p+1}-\mu^{p-1}\right) \bar{f}\left(\frac{b^{2 p}}{z a^{2 p-2}}\right), \\
& w_{i}=\frac{2}{(1+k)} \sum_{p=0}^{\infty} \mu^{p}\left\{f\left(\frac{z b^{2 p}}{a^{2 p}}\right)+\bar{f}\left(\frac{b^{2 p+2}}{z a^{2 p}}\right)\right\},
\end{aligned}
$$

where $\mu=(k-1) /(k+1)$.

1.30. The elliptic dielectric cylinder. The hydrodynamical problem of an elliptic cylinder in a general stream, and the analogous problem of the elliptic conductor in a general field, have long been solved. The case of the elliptic dielectric cylinder has, however, only been solved for certain specific external fields [7]. The difficulties arising from the singularities of transformation have been overcome here by using the results of 1.21 .

Consider the transformation

$$
z=\frac{c}{2}(\zeta+1 / \zeta), \quad z=c \sin w, w=u+i v
$$

Clearly the region outside the slit $A B$ in the $z$-plane transforms into the region outside the unit circle in the $\zeta$-plane. The ellipse $v=$ const. $=v_{1}$, say, of semi-axes $c \cosh v_{1}, c \sinh v_{1}$ in the $z$-plane, having $A, B$ as foci, becomes the circle $|\zeta|=e^{v_{1}}=a$, say. Let this ellipse be placed in the general field

$$
g(z) \equiv g\left\{\frac{c}{2}(\zeta+1 / \zeta)\right\}
$$

of which we consider the part

$$
h(\zeta)=\sum_{n=1}^{p} F_{n} \zeta^{n} \quad(p=\infty \text { is included })
$$

Split $h(\zeta)$ into

$$
G(\zeta)=(1 / 2)\{h(\zeta)+\bar{h}(\zeta)\} \text { and } H(\zeta)=(1 / 2)\{h(\zeta)-\bar{h}(\zeta)\} \text {, }
$$

so that $h(\zeta)=G(\zeta)+H(\zeta)$.

The component of $g(z)$ corresponding to $G(\zeta)$ will contain the real axis as a line of force, so that inside the ellipse, $A B$ will be a line of force. Thus the 
complex potentials due to $G(\zeta)$ can be found from $1.21(3)$, (4), by putting $b=1$ and $a=e^{v_{1}}$.

Now $g(z)$ gives rise to a field inside the dielectric ellipse such that the electrostatic potential is continuous in crossing $A B$. This means that in the $\zeta$-plane the values of the potential on $|\zeta|=1$ are the same at \pm am $\zeta$, and so the terms derived from $H(\zeta)$ can be found from $1.21(1),(2)$, again by putting $b=1$ and $a=e^{v_{1}}$.

Adding the complex potentials thus formed from $G(\zeta), H(\zeta)$, we find that the complex potentials outside and inside the ellipse are given respectively by

$$
\begin{aligned}
w_{0}=h(\zeta)-\mu \bar{h}\left(\frac{e^{2 v_{1}}}{\zeta}\right) & \\
& +\frac{4 k}{(1+k)^{2}}\left\{\sum_{p=0}^{\infty} \mu^{p} h\left(1 / \zeta e^{2 p v_{1}}\right)+\sum_{q=1}^{\infty} \mu^{q} \bar{h}\left(1 / \zeta e^{2 q v_{1}}\right)\right\},
\end{aligned}
$$

and

$$
\begin{aligned}
w_{i}=\frac{2}{(1+k)}\left\{\sum_{p=0}^{\infty} \mu^{p}\left\{h\left(\zeta / e^{2 p v_{1}}\right)+h\left(1 / \zeta e^{2 p v_{1}}\right)\right\}\right. \\
\quad+\frac{2}{(1+k)} \sum_{q=1}^{\infty} \mu^{q}\left\{\bar{h}\left(\zeta / e^{2 q v_{1}}\right)+\bar{h}\left(1 / \zeta e^{2 q v_{1}}\right)\right\},
\end{aligned}
$$

where $\sum$ refers to even powers, $\sum^{\prime}$ to odd powers, $\mu=(k-1) /(k+1)$, and $\zeta=i e^{v-i u}$.

Note that $w_{i}$ is of the form

$$
w_{i}=\sum_{n=1}^{p} A_{n}\left(\zeta^{n}+1 / \zeta^{n}\right)
$$

1.31. External line charge. Consider now a line charge $m$ at $\left(u_{0}, v_{0}\right)$ placed outside the elliptic dielectric cylinder parallel to the axis of the cylinder. In the $z$-plane we have

$$
g(z)=-2 m \log \left(z-z_{0}\right)=-2 m \log \left\{\frac{\left(\zeta-\zeta_{0}\right)\left(\zeta-1 / \zeta_{0}\right)}{\zeta}\right\},
$$


apart from an additive constant. We do not consider the terms

$$
-2 m \log \left(\zeta-1 / \zeta_{0}\right),-2 m \log \zeta
$$

since these refer to line charges inside the region $|\zeta|<1$ which corresponds to a different sheet on the Riemann surface.

Thus we take

$$
h(\zeta)=-2 m \log \left(\zeta-\zeta_{0}\right), \text { where } \zeta_{0}=i e^{v_{0}-i u_{0}}
$$

From 1.30 (3) we have

$$
w_{0}=-2 m \log \left(\zeta-\zeta_{0}\right)+2 m \mu \log \left(\frac{e^{2 v_{1}}}{\zeta}-\bar{\zeta}_{0}\right)
$$

(2)

$$
\begin{aligned}
&+\frac{4 k}{(1+k)^{2}}\left\{\sum_{p=0}^{\infty} \mu^{p}\left(-2 m \log \left(\frac{1}{\zeta e^{2 p v_{1}}}-\zeta_{0}\right)\right)\right. \\
&\left.+\sum_{q=1}^{\infty} \mu^{q}\left(-2 m \log \left(\frac{1}{\zeta e^{2 q v_{1}}}-\bar{\zeta}_{0}\right)\right)\right\} \\
&=-2 m \log \left\{1-e^{v-v_{0}-i\left(u-u_{0}\right)}\right\}+2 m \mu \log \left(1-e^{2 v_{1}-v-v_{0}+i\left(u-u_{0}\right)}\right)
\end{aligned}
$$

(3) $-\frac{8 m k}{(1+k)^{2}}\left\{\sum_{p=0}^{\infty} \mu^{p}\left\{\log \left(1+e^{i\left(u+u_{0}\right)-\left(v+v_{0}+2 p v_{1}\right)}\right)\right\}\right.$

$$
\left.+\sum_{q=1}^{\infty} \mu^{q}\left\{\log \left(1-e^{i\left(u-u_{0}\right)-\left(v+v_{0}+2 q v_{1}\right)}\right)\right\}\right\}
$$

neglecting any additive constants.

From 1.30 (4) we obtain the following result, where again we have neglected additive constants.

$$
\text { (4) } \begin{aligned}
w_{i}=\frac{2}{(1+k)} & \left\{\sum_{p=0}^{\infty} \mu^{p}\left\{-2 m \log \left(\zeta / e^{2 p v_{1}}-\zeta_{0}\right)-2 m \log \left(1 / \zeta e^{2 p v_{1}}-\zeta_{0}\right)\right\}\right. \\
& \left.+\sum_{q=1}^{\infty} \mu^{q}\left\{-2 m \log \left(\zeta / e^{2 q v_{1}}-\bar{\zeta}_{0}\right)-2 m \log \left(1 / \zeta e^{2 q v_{1}}-\bar{\zeta}_{0}\right)\right\}\right\}
\end{aligned}
$$


(5)

$$
\begin{gathered}
=-\frac{4 m}{(1+k)}\left\{\sum_{p=0}^{\infty} \mu^{p}\left\{\log 2\left[\sin (u+i v)-\sin \left(u_{0}+i\left(2 p v_{1}+v_{0}\right)\right)\right]\right\}\right. \\
\left.\quad+\sum_{q=1}^{\infty} \mu^{q}\left\{\log 2\left[\sin (u+i v)-\sin \left(u_{0}-i\left(2 q v_{1}+v_{0}\right)\right)\right]\right\}\right\} .
\end{gathered}
$$

1.32. Internal line-charge. By putting $a_{3, n}=0$ in the results of 1.20 , and proceeding exactly as in $1.21,1.30$, we obtain the external and internal fields due to a line charge $m$ at $\zeta=\zeta_{0}$ inside the dielectric elliptic cylinder $v_{1}$ in the form

$$
\begin{aligned}
& w_{0}=-\frac{4 m}{(1+\dot{k})}\left\{\sum_{p=0}^{\infty} \mu^{p} \log \left(\zeta-\zeta_{0} / e^{2 p v_{1}}\right)\left(\zeta-1 / e^{2 p v_{1}} \zeta_{0}\right)\right. \\
& \left.+\sum_{q=1}^{\infty} \mu^{q} \log \left(\zeta-\bar{\zeta}_{0} / e^{2 q v_{1}}\right)\left(\zeta-1 / e^{2 q v_{1}} \bar{\zeta}_{0}\right)\right\}+2 m \log \zeta \\
& u_{i}=-\frac{2 m}{k}\left\{\sum_{p=0}^{\infty} \mu^{p} \log \left(\zeta-1 / e^{2 p v_{1}} \zeta_{0}\right)\right. \\
& +\sum_{p=0}^{\infty} \mu^{p+1} \log \left(\zeta-\bar{\zeta}_{0} / e^{2(p+1) v_{1}}\right)\left(\zeta-e^{2(p+1) v_{1}} / \bar{\zeta}_{0}\right)\left(\zeta-e^{2(p+1) v_{1}} \bar{\zeta}_{0}\right) \\
& +\sum_{q=1}^{\infty}{ }^{q}{ }^{q} \log \left(\zeta-1 / e^{2 q v_{1}} \bar{\zeta}_{0}\right) \\
& \left.+\sum_{q=1}^{\infty} \mu^{q+1} \log \left(\zeta-\zeta_{0} / e^{2(q+1) v_{1}}\right)\left(\zeta-e^{2(q+1) v_{1}} / \zeta_{0}\right)\left(\zeta-e^{2(q+1) v_{1}} \zeta_{0}\right)\right\} \\
& -\frac{2 m}{k} \log \left(\zeta-\zeta_{0}\right)+2 m \log \zeta
\end{aligned}
$$


The fields due to line doublets both inside and outside the elliptic cylinder can be deduced.

1.40. Force and couple on the elliptic cylinder. Let the cylinder be placed in the general field

$$
g(z)=\sum_{n=1}^{\infty} E_{n} z^{n}
$$

Then

$$
z=\frac{c}{2}(\zeta+1 / \zeta)
$$

transforms $g(z)$ into a function of $\zeta$, of which we consider

$$
h(\zeta)=\sum_{n=1}^{\infty} F_{n} \zeta^{n}
$$

The field inside the cylinder, being of the form given by 1.30 (5), will contribute nothing to either the complex force or couple of 1.1.

From 1.30 ( 3 ) we see that

$$
w_{0}=\sum_{n=1}^{\infty} F_{n} \zeta^{n}+\sum_{n=1}^{\infty} G_{n} / \zeta^{n}
$$

where

$$
G_{n}=\frac{a^{4 n} F_{n}\left(\mathrm{i}-\mu^{2}\right)+a^{2 n} \mu \bar{F}_{n}\left(1-a^{4 n}\right)}{a^{4 n}-\mu^{2}}, a=e^{v_{1}}
$$

The complex force. This is given by

$$
Y+i X=\frac{1}{8 \pi} \int_{C}\left(\frac{d w_{0}}{d \zeta}\right)^{2} \frac{d \zeta}{d z / d \zeta}=\frac{1}{4 \pi c} \int_{C}\left\{\sum_{n=1}^{\infty} n F_{n} \zeta^{n}-\sum_{n=1}^{\infty} n G_{n} / \zeta^{n}\right\}^{2} \frac{d \zeta}{\left(\zeta^{2}-1\right)}
$$


There are poles at $\zeta=0, \zeta=1, \zeta=-1$, and so

$$
Y+i X=\frac{i}{c} \sum_{r=2}^{\infty} \sum_{s=1}^{\infty} r s F_{r}\left(F_{s}-G_{s}\right), r>s, r-s \text { odd. }
$$

The couple. This is given by

$$
\begin{aligned}
\Gamma & =R \frac{1}{8 \pi} \int_{C}\left(\frac{d w_{0}}{d \zeta}\right)^{2} \frac{z d \zeta}{d z / d \zeta} \\
& =R \frac{1}{8 \pi} \int_{C}\left\{\sum_{n=1}^{\infty} n F_{n} \zeta^{n}-\sum_{n=1}^{\infty} n G_{n} / \zeta^{n}\right\}^{2} \frac{\left(\zeta^{2}+1\right) d \zeta}{\left(\zeta^{2}-1\right) \zeta}
\end{aligned}
$$

Again there are poles at $\zeta=0, \zeta=1, \zeta=-1$, and so

(5) $\Gamma=R\left\{\frac{i}{2} \sum_{n=1}^{\infty} n^{2} F_{n}\left(F_{n}-G_{n}\right)+i \sum_{r=3}^{\infty} \sum_{s=1}^{\infty} r s F_{r}\left(F_{s}-G_{s}\right)\right\}$,

$$
r>s, r-s \text { even. }
$$

1.41. Force and couple due to external line charge. The perturbed potentials are given in 1.31 , and we note that the internal field will contribute nothing to either the force or the couple. Thus the complex force is

$$
\begin{aligned}
& Y+i X=\frac{1}{8 \pi} \int_{C} \frac{2 \zeta^{2}}{c\left(\zeta^{2}-1\right)}\left\{2 m \left[-\frac{1}{\zeta-\zeta_{0}}-\frac{\mu a^{2}}{\zeta\left(a^{2}-\bar{\zeta}_{0} \zeta\right)}\right.\right. \\
& \left.\left.+\frac{4 k}{(1+k)^{2}}\left\{\sum_{p=0}^{\infty} \frac{\mu^{p}}{\zeta\left(1-a^{2 p} \zeta_{0} \zeta\right)}+\sum_{q=1}^{\infty} \cdot \frac{\mu^{q}}{\zeta\left(1-a^{2 q} \bar{\zeta}_{0} \zeta\right)}\right]\right]\right\}^{2} d \zeta .
\end{aligned}
$$

To evaluate this integral we draw a circle $S$ of large radius to enclose both the cylinder $C$ and the point $\zeta=\zeta_{0}$. Thus we have

$$
\frac{1}{8 \pi} \int_{C}[] d \zeta=\frac{1}{8 \pi} \int_{S}[] d \zeta-\frac{1}{8 \pi} \int_{\gamma}[] d \zeta,
$$


where $\gamma$ is a small contour drawn around $\zeta=\zeta_{0}$.

To determine the integral around $S$, we expand in powers of $1 / \zeta$, and the integral around $\gamma$ is obtained in the usual manner. Carrying out these integrations we obtain

$$
\begin{aligned}
& Y+i X=\frac{4 m^{2} i \zeta_{0}}{c\left(\zeta_{0}^{2}-1\right)}\left\{-\frac{\mu a^{2}}{\left(a^{2}-\zeta_{0} \bar{\zeta}_{0}\right)}+\frac{1}{\left(\zeta_{0}^{2}-1\right)}\right. \\
& \left.\quad+\frac{4 k}{(1+k)^{2}}\left[\sum_{p=0}^{\infty} \frac{\mu^{p}}{\left(1-a^{2 p} \zeta_{0}^{2}\right)}+\sum_{q=1}^{\infty} \frac{\mu^{q}}{\left(1-a^{2 q} \zeta_{0} \bar{\zeta}_{0}\right)}\right]\right\}
\end{aligned}
$$

In a similar manner, the couple is given by

(2)

$$
\begin{aligned}
\Gamma=R \frac{2 i m^{2}\left(\zeta_{0}^{2}+1\right)}{\left(\zeta_{0}^{2}-1\right)}\left\{-\frac{\mu a^{2}}{\left(a^{2}-\zeta_{0} \overline{\zeta_{0}}\right)}+\frac{1}{\left(\zeta_{0}^{2}-1\right)}\right. \\
\left.+\frac{4 k}{(1+k)^{2}}\left[\sum_{p=0}^{\infty} \frac{\mu^{p}}{\left(1-a^{2 p} \zeta_{0}^{2}\right)}+\sum_{q=1}^{\infty} \cdot \frac{\mu^{q}}{\left(1-a^{2 q} \zeta_{0} \overline{\zeta_{0}}\right)}\right]\right\} .
\end{aligned}
$$

These results could equally well have been obtained by the use of Lagally's The orem [2].

By a similar method the resultant force and couple on the elliptic cylinder can be evaluated for an internal line charge.

1.50. Elliptic conducting cylinder with confocal dielectric sheath. Let $v=v_{2}$ be a conducting elliptic cylinder, surrounded by a confocal elliptic sheath $v=v_{1}$ of dielectric constant $k$. If this system is placed in a general field $g(z)$ in air, then we consider, as usual, the unperturbed portion $h(\zeta)$, and $1.21(1),(2)$, give the complex potentials outside and inside the dielectric in the form

$$
\begin{aligned}
& w_{0}=h(\zeta)+\lambda \bar{h}\left(\frac{e^{2 v_{1}}}{\zeta}\right)+\sum_{p=1}^{\infty}\left(\lambda^{p+1}-\lambda^{p-1}\right) \bar{h}\left(\frac{e^{2 p v_{2}}}{\zeta e^{2(p-1) v_{1}}}\right), \\
& w_{i}=\frac{2}{(1+k)} \sum_{p=0}^{\infty} \lambda^{p}\left\{h\left(\frac{\zeta e^{2 p v_{2}}}{e^{2 p v_{1}}}\right)-\bar{h}\left(\frac{e^{2(p+1) v_{2}}}{e^{2 p v_{1}} \zeta}\right)\right\} .
\end{aligned}
$$


If $h(\zeta)$ is of the form

$$
h(\zeta)=\sum_{n=1}^{\infty} F_{n} \zeta^{n}
$$

then

$$
w_{0}=\sum_{n=1}^{\infty} F_{n} \zeta^{n}+\sum_{n=1}^{\infty} \frac{G_{n}}{\zeta^{n}}
$$

where

$$
G_{n}=-\frac{\bar{F}_{n} a^{2 n}\left\{b^{2 n}(k+1)+a^{2 n}(k-1)\right\}}{\left\{a^{2 n}(k+1)+b^{2 n}(k-1)\right\}},
$$

and $a=e^{v_{1}}, b=e^{v_{2}}$.

Also we have

$$
w_{i}=\sum_{n=1}^{\infty} H_{n} \zeta^{n}+\sum_{n=1}^{\infty} K_{n} / \zeta^{n},
$$

where

(6) $H_{n}=\frac{2 a^{2 n} F_{n}}{\left\{a^{2 n}(k+1)+b^{2 n}(k-1)\right\}}, K_{n}=-\frac{2 a^{2 n} b^{2 n} \bar{F}_{n}}{\left\{a^{2 n}(k+1)+b^{2 n}(k-1)\right\}}$.

Force on the conductor. The only contribution comes from $w_{i}$, which as in 1.40 gives

$$
Y+i X=\frac{i k}{c} \sum_{r=2}^{\infty} \sum_{s=1}^{\infty} r s H_{r}\left(H_{s}-K_{s}\right), r>s, r-s \text { odd } .
$$

Couple on the conductor. Similarly we have

(8) $\Gamma=R\left\{\frac{i k}{2}\left[\sum_{n=1}^{\infty} n^{2} H_{n}\left(H_{n}-K_{n}\right)\right]+i k\left[\sum_{r=3}^{\infty} \sum_{s=1}^{\infty} r s H_{r}\left(H_{s}-K_{s}\right)\right]\right\}$, 
Force on the dielectric boundary. Both $w_{0}, w_{i}$ contribute, giving

(9) $Y+i X=\frac{i}{c} \sum_{r=2}^{\infty} \sum_{s=1}^{\infty} r s F_{r}\left(F_{s}-G_{s}\right)-\frac{i k}{c} \sum_{r=2}^{\infty} \sum_{s=1}^{\infty} r s H_{r}\left(H_{s}-K_{s}\right)$,

$$
r>s, r-s \text { odd. }
$$

Couple on the dielectric boundary. In a similar manner, we see that

$$
\begin{aligned}
\Gamma & =R\left\{\frac{i}{2}\left[\sum_{n=1}^{\infty} n^{2} F_{n}\left(F_{n}-G_{n}\right)\right]+i\left[\sum_{r=3}^{\infty} \sum_{s=1}^{\infty} r s F_{r}\left(F_{s}-G_{s}\right)\right]\right\} \\
& -\operatorname{Ri}\left\{\frac{i k}{2}\left[\sum_{n=1}^{\infty} n^{2} H_{n}\left(H_{n}-K_{n}\right)\right]+i k\left[\sum_{r=3}^{\infty} \sum_{s=1}^{\infty} r s H_{r}\left(H_{s}-K_{s}\right)\right]\right\},
\end{aligned}
$$

$$
r>s, r-s \text { even }
$$

1.51. Elliptic dielectric cylinder with confocal dielectric sheath. Let now $v=v_{2}$ be an elliptic cylinder of dielectric constant $k_{1}$, while between $v=v_{2}$ and $v=v_{1}$ is dielectric material of constant $k_{2}$, and suppose that this system is placed in a medium of dielectric constant $k_{3}$, in which there is a field whose unperturbed form is $g(z)$, of which we consider the portion

$$
h(\zeta)=\sum_{n=1}^{\infty} F_{n} \zeta^{n}
$$

Equation $1.30(5)$ gives the form of the field inside $v_{2}$, and so we see that the perturbed potentials will be given by

$$
\begin{aligned}
& w_{1}=\sum_{n=1}^{\infty} a_{1, n}\left(\zeta^{n}+1 / \zeta^{n}\right), \\
& w_{2}=\sum_{n=1}^{\infty} a_{2, n} \zeta^{n}+\sum_{n=1}^{\infty} b_{2, n} \zeta^{-n}, w_{3}=\sum_{n=1}^{\infty} F_{n} \zeta^{n}+\sum_{n=1}^{\infty} b_{3, n} \zeta^{-n} .
\end{aligned}
$$


The boundary conditions of 1.20 yield

$$
\begin{gathered}
{\left[b^{4 n} \alpha_{n}^{2}-\beta_{n}^{2}\right] a_{1, n}=4 k_{3} k_{2} a^{2 n} b^{2 n}\left\{b^{2 n} \alpha_{n} F_{n}-\beta_{n} \bar{F}_{n}\right\},} \\
{\left[b^{4 n} \alpha_{n}^{2}-\beta_{n}^{2}\right] a_{2, n}=2 k_{3} a^{2 n}\left\{\left[b^{4 n}\left(k_{2}+k_{1}\right) \alpha_{n}-\left(k_{2}-k_{1}\right) \beta_{n}\right] F_{n}\right.} \\
\left.-\left[b^{2 n}\left(k_{2}+k_{1}\right) \beta_{n}-b^{2 n}\left(k_{2}-k_{1}\right) \alpha_{n}\right] \bar{F}_{n}\right\}, \\
{\left[b^{4 n} \alpha_{n}^{2}-\beta_{n}^{2}\right] b_{2, n}=2 k_{3} a^{2 n} b^{2 n}\left\{\left[b^{4 n}\left(k_{2}-k_{1}\right) \alpha_{n}-\left(k_{2}+k_{1}\right) \beta_{n}\right] \bar{F}_{n}\right.} \\
\left.-\left[b^{2 n}\left(k_{2}-k_{1}\right) \beta_{n}-b^{2 n}\left(k_{2}+k_{1}\right) \alpha_{n}\right] F_{n}\right\}, \\
{\left[b^{4 n} \alpha_{n}^{2}-\beta_{n}^{2}\right] b_{3, n}=\left\{b^{4 n} a^{2 n}\left[a^{2 n}\left(k_{2}+k_{1}\right)\left(k_{3}-k_{2}\right)+b^{2 n}\left(k_{2}-k_{1}\right)\left(k_{3}+k_{2}\right)\right] \alpha_{n}\right.} \\
\left.-a^{2 n}\left[a^{2 n}\left(k_{3}-k_{2}\right)\left(k_{2}-k_{1}\right)+b^{2 n}\left(k_{3}+k_{2}\right)\left(k_{2}+k_{1}\right)\right] \beta_{n}\right\} \bar{F}_{n} \\
-\left\{a^{2 n} b^{2 n}\left[a^{2 n}\left(k_{3}-k_{2}\right)\left(k_{2}+k_{1}\right)+b^{2 n}\left(k_{3}+k_{2}\right)\left(k_{2}-k_{1}\right)\right] \beta_{n}\right. \\
\left.-a^{2 n} b^{2 n}\left[a^{2 n}\left(k_{3}-k_{2}\right)\left(k_{2}-k_{1}\right)+b^{2 n}\left(k_{3}+k_{2}\right)\left(k_{2}+k_{1}\right)\right] \alpha_{n}\right\} F_{n},
\end{gathered}
$$

where

$$
\begin{aligned}
& a_{n}=a^{2 n}\left(k_{3}+k_{2}\right)\left(k_{2}+k_{1}\right)+b^{2 n}\left(k_{3}-k_{2}\right)\left(k_{2}-k_{1}\right), \\
& \beta_{n}=a^{2 n}\left(k_{3}+k_{2}\right)\left(k_{2}-k_{1}\right)+b^{2 n}\left(k_{3}-k_{2}\right)\left(k_{2}+k_{1}\right), a=e^{v_{1}}, b=e^{v_{2}} .
\end{aligned}
$$

To evaluate the force and couple on each dielectric boundary, we proceed exactly as in 1.50 .

1.6. Parabolic dielectric cylinder. W'e can use results already obtained to get the internal and external perturbed fields due to the presence of a parabolic dielectric cylinder of constant $k$.

The transformations

$$
z=t^{2}, \zeta=e^{-i t},
$$

where 


$$
z=x+i y, t=\alpha+i \beta, \quad \zeta=\xi+i \eta
$$

are such that $\beta=$ const. $=\beta_{1}$, say, gives a parabola in the $z$-plane which becomes a circle of radius $e^{\beta_{1}}$ in the $\zeta$-plane. The limits of $c_{1}, \beta$ are given respectively by

$$
-\infty<\alpha<\infty \text { and } 0<\beta<\infty
$$

The limiting case $\beta=0$ becomes the positive $x$-axis in the $z$-plane, and the unit circle in the $\zeta$-plane.

As an example, let us find the fields due to an external line charge $m$ placed parallel to the generators of the cylinder at $\left(\alpha_{0}, \beta_{0}\right)$. We have

$$
h(t)=-2 m \log \left(t-t_{0}\right), t_{0}=\alpha_{0}+i \beta_{0},
$$

and so we obtain

$$
\begin{aligned}
& w_{0}=-2 m \log \left(t-t_{0}\right)+2 m \mu \log \left(t-\overline{t_{0}}-2 i \beta_{1}\right) \\
& -\frac{8 m k}{(1+k)^{2}}\left\{\sum_{p=0}^{\infty} \mu^{p} \log \left(t+t_{0}+2 i p \beta_{1}\right)+\sum_{q=1}^{\infty} \mu^{q} \log \left(t-\overline{t_{0}}+2 i q \beta_{1}\right)\right\},
\end{aligned}
$$

and

(3)

$$
\begin{aligned}
w_{i}=-\frac{4 m}{(1+k)}\left\{\sum_{p=0}^{\infty} \mu^{p} \log \left(t-t_{0}-2 i p \beta_{1}\right)\left(t+t_{0}+2 i p \beta_{1}\right)\right. \\
\\
\left.+\sum_{q=1}^{\infty} \mu^{q} \log \left(t+\bar{t}_{0}-2 i q \beta_{1}\right)\left(t-\bar{t}_{0}+2 i q \beta_{1}\right)\right\}
\end{aligned}
$$

1.7. Dielectric annulus and dielectric slab. If, in the results of 1.20 , we set

$$
k_{1}=k_{3}=1, k_{2}=k, a_{3, n}=E_{n}, m=0 \text {, }
$$

we get the elementary case of an annulus of dielectric constant $k$. If this annulus is placed in the general field $g(z)$, then the perturbed fields are seen to be

$$
w_{1}=\frac{4 k}{(1+k)^{2}} \sum_{p=0}^{\infty} \lambda^{2 p} g\left(\frac{b^{2 p} z}{a^{2 p}}\right) \text {, }
$$




$$
\begin{aligned}
& w_{2}=\frac{2}{(1+k)}\left\{\sum_{p=0}^{\infty} \lambda^{2 p} g\left(\frac{b^{2 p} z}{a^{2 p}}\right)-\sum_{p=0}^{\infty} \lambda^{2 p+1} \bar{g}\left(\frac{b^{2(p+1)}}{a^{2 p} z}\right)\right\}, \\
& w_{3}=g(z)+\sum_{p=0}^{\infty} \lambda^{2 p+1}\left\{\bar{g}\left(\frac{b^{2 p}}{a^{2(p-1)} z}\right)-\bar{g}\left(\frac{b^{2(p+1)}}{a^{2 p} z}\right)\right\} .
\end{aligned}
$$

If now we put $z=e^{-i t}$, where $t=a+i \beta$, we can transform the dielectric annulus into a dielectric slab. Setting $b=1, a=e^{\beta_{1}},\left(\beta_{1}\right.$ positive ), in (1), (2), (3) above we get

$$
w_{1}=\frac{4 k}{(1+k)^{2}} \sum_{p=0}^{\infty} \lambda^{2 p} f\left(t-2 i p \beta_{1}\right)
$$

$$
w_{2}=\frac{2}{(1+k)}\left\{\sum_{p=0}^{\infty} \lambda^{2 p} f\left(t-2 i p \beta_{1}\right)-\sum_{p=0}^{\infty} \lambda^{2 p+1} \bar{f}\left(t+2 i p \beta_{1}\right)\right\}
$$

$$
w_{3}=f(t)+\sum_{p=0}^{\infty} \lambda^{2 p+1}\left\{\bar{f}\left(t+2 i(p-1) \beta_{1}\right)-\bar{f}\left(t+2 i p \beta_{1}\right)\right\}
$$

as the perturbed fields in the parts of the $t$-plane given respectively by

$$
0 \geq \beta, \beta_{1} \geq \beta \geq 0, \beta \geq \beta_{1},
$$

when the unperturbed field $f(t)$ is caused by singularities placed in the region $\beta \geq \beta_{1}$.

It can easily be verified that $(4),(5),(6)$ above satisfy the required boundary conditions in the $t$-plane, which are

$$
R\left(w_{1}\right)=R\left(w_{2}\right), d\left(w_{1}\right)=k d\left(w_{2}\right) \text { when } \beta=0 \text {, }
$$

and

$$
R\left(w_{2}\right)=R\left(w_{3}\right), k d\left(w_{2}\right)=d\left(w_{3}\right) \text { when } \beta=\beta_{1} \text {. }
$$

Moreover, since no new singularities are introduced into the regions concerned, these formulae can conveniently be used to obtain image systems. 


\section{ThreE-Dimensional FieldS}

2.1. Mechanical force and couple. The components of resultant force and couple corresponding to the Blasius type formulae of 1.1 have leen given previously [4], and will not be discussed further in this paper.

2.20. The sphere theorem. It has been shown [3] that if a dielectric sphere of radius $a$, dielectric constant $k_{i}$, is placed with its center at the origin of coordinates, in a nedium of constant $k_{0}$ in which there is a general field whose undisturbed potential is $\phi\left(x_{*} y, z\right)$, having no singularities inside or on $r=a$, then the perturbed potentials inside and outside the sphere are respectively

$$
\phi_{i}=\frac{2}{(1+k)} \phi(x, y, z)+\frac{(k-1)}{(k+1)^{2}} \int_{0}^{1} t^{-k /(k+1)} \phi(x t, y t, z t) d t,
$$

and

$$
\begin{aligned}
\phi_{0}=\phi(x, y, z)-\frac{(k-1)}{(k+1)} & \frac{a}{r} \phi\left(x_{1}, y_{1}, z_{1}\right)+ \\
& +\frac{(k-1)}{(k+1)^{2}} \frac{a}{r} \int_{0}^{1} t^{-k /(k+1)} \phi\left(x_{1} t, y_{1} t_{2} z_{1} t\right) d t,
\end{aligned}
$$

where

$$
x_{1}=\frac{a^{2} x}{r^{2}}, y_{1}=\frac{a^{2} y}{r^{2}}, z_{1}=\frac{a^{2} z}{r^{2}}, r^{2}=x^{2}+y^{2}+z^{2}, \text { and } k=k_{i} / k_{0} .
$$

Similarly, we can show that if there is a field whose undisturbed potential $\phi(x, y, z)$ is caused only by singularities placed inside the sphere $r=a$, whose dielectric constant is $k_{i}$, and if this sphere is surrounded by dielectric material of constant $k_{0}$, the perturbed potentials are

$$
\begin{aligned}
\phi_{0}=\frac{2}{\left(k^{\prime}+1\right)} & \phi(x, y, z)+ \\
& +\frac{\left(k^{\prime}-1\right)}{\left(k^{\prime}+1\right)^{2}} \int_{\infty}^{1} t^{-k^{\prime} /\left(k^{\prime}+1\right)} \phi(x t, y t, z t) d t,
\end{aligned}
$$

and 


$$
\begin{aligned}
\phi_{i}=\phi(x, y, z) & -\frac{\left(k^{\prime}-1\right)}{\left(k^{\prime}+1\right)} \frac{a}{r} \phi\left(x_{1}, y_{1}, z_{1}\right) \\
& +\frac{\left(k^{\prime}-1\right)}{\left(k^{\prime}+1\right)^{2}} \frac{a}{r} \int_{\infty}^{1} t^{-k^{\prime} /\left(k^{\prime}+1\right)} \phi\left(x_{1} t, y_{1} t, z_{1} t\right) d t,
\end{aligned}
$$

where $k^{\prime}=k_{0} / k_{i}$.

2.21. Application of Stokes' stream function for axisymmetric fields. We now consider axisymmetric fields given in terms of the spherical polar coordinates $r, \theta$. Stokes' stream function [6] $\dot{\psi}(r, \theta)$ satisfies the equation

$$
\left\{r^{2} \frac{\partial^{2}}{\partial r^{2}}+\sin \theta \frac{\partial}{\partial \theta}\left(\frac{1}{\sin \theta} \frac{\partial}{\partial \theta}\right)\right\} \psi(r, \theta)=0,
$$

and is related to the potential $\phi$ by

$$
\frac{\partial \psi}{\partial \mu}=-r^{2} \frac{\partial \phi}{\partial r}, \frac{\partial \psi}{\partial r}=\left(1-\mu^{2}\right) \frac{\partial \phi}{\partial \mu},
$$

where $\mu=\cos \theta$. Thus to the solutions

$$
r^{n} P_{n} \text { and } r^{-(n+1)} P_{n}
$$

of Laplace's equation correspond respectively

$$
\frac{\left(1-\mu^{2}\right)}{(n+1)} r^{n+1} \frac{d P_{n}}{d \mu} \text { and }-\frac{\left(1-\mu^{2}\right)}{n} r^{-n} \frac{d P_{n}}{d \mu} .
$$

The perturbed stream functions inside and outside the dielectric sphere of 2.20 are thus given by

$$
\psi_{i}=\left(1-\mu^{2}\right) \sum_{n=0}^{\infty} \frac{(2 n+1) E_{n} r^{n+1}}{(n+1)\{n(k+1)+1\}} \frac{d P_{n}}{d \mu}
$$

and

$$
\psi_{0}=\psi(r, \theta)-\left(1-\mu^{2}\right) \sum_{n=0}^{\infty} \frac{(1-k) a^{2 n+1} E_{n}}{\{n(k+1)+1\} r^{n}} \frac{d P_{n}}{d \mu}
$$


where the unperturbed external electrostatic potential is taken to be

$$
\phi=\sum_{n=0}^{\infty} E_{n} r^{n} P_{n}
$$

with the corresponding stream function

$$
\psi=\left(1-\mu^{2}\right) \sum_{n=0}^{\infty} \frac{E_{n} r^{n+1}}{(n+1)} \frac{d P_{n}}{d \mu} .
$$

By manipulating the coefficients as in the proof of the Sphere Theorem [3], we can put these stream functions in terms of the unperturbed stream function in the form

$$
\psi_{i}=\frac{2}{(1+k)} \psi(r, \theta)+\frac{(k-1)}{(k+1)^{2}} \int_{0}^{1} t^{-(2 k+1) /(k+1)} \psi(r t, \theta) d t,
$$

$$
\begin{aligned}
\psi_{0}=\psi(r, \theta) & -\frac{(1-k)}{(1+k)} \frac{r}{a} \psi\left(\frac{a^{2}}{r}, \theta\right) \\
& -\frac{k(1-k)}{(1+k)^{2}} \frac{r}{a} \int_{0}^{1} t^{-(2 k+1) /(k+1)} \psi\left(\frac{a^{2} t}{r}, \theta\right) d t,
\end{aligned}
$$

where, as before, $k=k_{i} / k_{0}$.

In a similar manner, the perturbed stream. functions for an axisymmetric field caused by singularities inside the dielectric sphere can be established in the form

$$
\psi_{0}=\frac{2}{\left(k^{\prime}+1\right)} \psi(r, \theta)+\frac{\left(k^{\prime}-1\right)}{\left(k^{\prime}+1\right)^{2}} \int_{\infty}^{1} t^{-\left(2 k^{\prime}+1\right) /\left(k^{\prime}+1\right)} \psi(r t, \theta) d t,
$$

$$
\begin{aligned}
\psi_{i}=\psi(r, \theta) & -\frac{\left(1-k^{\prime}\right)}{\left(1+k^{\prime}\right)} \frac{r}{a} \psi\left(\frac{a^{2}}{r}, \theta\right) \\
& -\frac{k^{\prime}\left(1-k^{\prime}\right)}{\left(1+k^{\prime}\right)^{2}} \frac{r}{a} \int_{\infty}^{1} t^{-\left(2 k^{\prime}+1\right) /\left(k^{\prime}+1\right)} \psi\left(\frac{a^{2} t}{r}, \theta\right) d t,
\end{aligned}
$$


where $k^{\prime}=k_{0} / k_{i}$.

For the hydrodynamical case of an external axisymmetric field in the presence of a sphere we set $k=0$ in (4), thus obtaining the perturbed stream function in the simple form

$$
\Psi=\psi(r, \theta)-\frac{r}{a} \psi\left(\frac{a^{2}}{r}, \theta\right)
$$

The result (7) also holds for fluid motion occupying the region $r \leq a$, when $r=a$ is made a rigid boundary. Examples have been given elsewhere [1].

The chief advantage of these formulae is that the "flow-sheets" are given directly. However, the results are not so general as those of 2.20 , and the functional form is no simpler, except in the hydrodynamical case (7) where no integral term is involved.

\section{REFERENCE}

1. S. F.J. Butler, A note on Stokes' stream function for motion with a spherical boundary, Proc. Cambridge Philos. Soc. 49 Pt. I (1953), 169.

2. L. M. Milne-Thomson, Theoretical hydrodynamics, Macmillan, London, 1949, 208.

3. G. Power, Change in potential due to a dielectric sphere, Amer. Math. Monthly 58 (1951), 249.

4. 233-246.

, Forces on the boundary of a dielectric, Pacific J. Math. 3 (1953),

5. —, A dielectric cylinder, Math. Gazette, Vol. XXXVII, No. 321, 220.

6. A.S. Ramsay, A treatise on hydromechanics, Part II Hydrodynamics, G. Bell and Sons, Ltd. London 1942, 164-167.

7. W. R. Smythe, Static and dynamic electricity, McGraw-Hill Book Company Inc., 2nd edition, 95-98, 108 example 53.

\section{UNIVERSITY OF NOTTINGHAM}




\title{
PACIFIC JOURNAL OF MATHEMATICS
}

\section{EDITORS}

\author{
M. M. SCHIFFER* \\ Stanf ord University \\ Stanf ord, California \\ E. HewitT \\ University of Washington \\ Seattle 5, Washington
}

\author{
P. P. DILWCR TH \\ California Institute of Technology \\ Pasadena 4, California \\ E. F. BECKENBACH** \\ University of California \\ Los Angeles 24, California
}

\section{ASSOCIATE EDITORS}

$\begin{array}{ll}\text { H. BUSEMANN } & \text { P. R. HALMOS } \\ \text { HERDERT FF.DERER } & \text { HEINZ HOPF } \\ \text { MARSH ALLL IHALL } & \text { R. D. JAMES }\end{array}$

MARSH AL.L II ALL

\author{
R. D. JAMES
}
BORGE JESSEN
PAUL LÉVY
GEORGE PÓLYA

\author{
J. J. STOKER \\ E. G. STRAUS
}
KÖSAKU YOSIDA

\section{SPONSORS}

UNIVERSITY OF BRITISH COLUMBIA CAI IFORNIA ENSTITUTE OF TECHNOLOGY UNIVERSITY OF CALIFORNIA, BERKELEY UNIVERSITY OF CALIFORNIA, DAVIS UNIVERSITY OF CALIFORNIA, LOS ANGELES UNIVERSITY OF CALIFORNI A, SANTA BARBARA UNIVERSITY OF NEVADA OREGON STATE COLLEGE UNIVERSITY OF OREGON
UNIVERSITY OF SOU THERN CALIFORNIA STANFORD RESE.ARCH INSTITUTE STANFORD UNIVERSITY WASHING TON STATE COLLEGE UNIVERSITY OF WASHINGTON

AMERICAN MATHEMATICAL SOCIETY HUGHES AIRCRAFT COMPANY SHELL DEVELOPMENT COMPANY

* To be succeeded in 1955, by H.L. Royden, Stanford University, Stanford, California.

${ }^{* *}$ To be succeeded in 1955, by E.G. Straus, University of California, Los Angeles 24, Calif.

\author{
Vari-Type Composition by \\ Elaine Barth
}

Printed in the United States of America by

Edwards Brothers, Inc., Ann Arbor, Michigan

UNIVERSITY OF CALIFORNIA PRESS * BERKELEY AND LOS ANGELES COPYRIGHT 1954 BY PACIFIC JOURNAL OF MATHEMATICS 


\section{Pacific Journal of Mathematics}

\section{Vol. 4, No. 1 \\ May, 1954}

Hugh D. Brunk, On the growth of functions having poles or zeros on the positive real axis ................................. 1

J. Copping, Application of a theorem of Pólya to the solution of an infinite

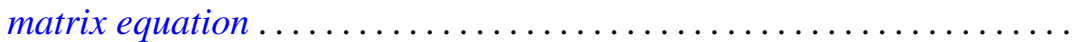

James Richard Jackson, On the existence problem of linear programming ................................... 29

Victor Klee, Invariant extension of linear functionals ............... 37

Shu-Teh Chen Moy, Characterizations of conditional expectation as a transformation on function spaces ..................... 47

Hukukane Nikaidô, On von Neumann's minimax theorem .............. 65

Gordon Marshall Petersen, Methods of summation .................. 73

G. Power, Some perturbed electrostatic fields .................. 79

Murray Harold Protter, The two noncharacteristic problem with data partly

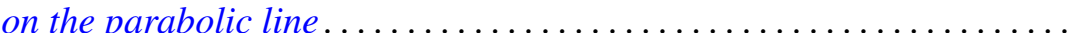

S. E. Rauch, Mapping properties of Cesàro sums of order two of the geometric series........................................... 109

Gerson B. Robison, Invariant integrals over a class of Banach spaces . . . . 123

Richard Steven Varga, Eigenvalues of circulant matrices .............. 151 